\title{
Scanning a photonic crystal slab nanocavity by condensation of xenon
}

\author{
S. Mosor, J. Hendrickson, ${ }^{\text {a) }}$ B. C. Richards, J. Sweet, G. Khitrova, and H. M. Gibbs \\ Optical Sciences Center, The University of Arizona, Tucson, Arizona 85721 \\ T. Yoshie \\ Department of Electrical and Computer Engineering, Duke University, Durham, North Carolina 27708 \\ A. Scherer \\ Department of Electrical Engineering, California Institute of Technology, Pasadena, California 91125 \\ O. B. Shchekin and D. G. Deppe \\ Microelectronics Research Center, Department of Electrical and Computer Engineering, \\ The University of Texas at Austin, Austin, Texas 78712
}

(Received 15 July 2005; accepted 25 August 2005; published online 28 September 2005)

\begin{abstract}
Allowing xenon or nitrogen gas to condense onto a photonic crystal slab nanocavity maintained at $10-20 \mathrm{~K}$ results in shifts of the nanocavity mode wavelength by as much as $5 \mathrm{~nm}(\cong 4 \mathrm{meV})$. This occurs in spite of the fact that the mode defect is achieved by omitting three holes to form the spacer. This technique should be useful in changing the detuning between a single quantum dot transition and the nanocavity mode for cavity quantum electrodynamics experiments, such as mapping out a strong coupling anticrossing curve. Compared with temperature scanning, it has a much larger scan range and avoids phonon broadening. (C) 2005 American Institute of Physics.
\end{abstract}

[DOI: $10.1063 / 1.2076435$ ]

Radiative coupling between a single quantum dot (SQD) and a small volume cavity alters the emission properties of the coupled system. In the weak coupling regime, the spontaneous emission has a single emission frequency, and it irreversibly escapes from the cavity. The SQD radiative emission rate is enhanced by the Purcell factor $F_{P}=3 \lambda^{3} Q /\left(4 \pi^{2} V\right)$ compared with the cavityless radiative emission rate $\gamma_{0} ; Q$ and $V$ are the quality factor and volume of the cavity, respectively, and $\lambda=\lambda_{0} / n$ is the wavelength of the light in the material with refractive index $n$. The several single-photon-on-demand sources that have been reported operate in the weak coupling regime. ${ }^{1-5}$ In the strong coupling regime, the Rabi frequency rate of exchange of the excitation between the SQD and the cavity, $g=\mu E_{\mathrm{vac}} / \hbar$, exceeds both the photon escape rate $\kappa$ and SQD dephasing rate $\gamma$. In the formula for $g$, it is assumed that the SQD has dipole moment $\mu$ and is in the peak of the root-mean-square intracavity field $E_{\mathrm{vac}}$ satisfying $\varepsilon_{0} n^{2}\left|E_{\mathrm{vac}}\right|^{2} V=h \nu / 2 ; \varepsilon_{0}$ is the permittivity of vacuum, and $\nu$ is the frequency of the cavity mode. The strong coupling makes the spontaneous emission reversible, i.e., a photon emitted by the excited SQD has a higher probability of being reabsorbed than escaping the cavity. For zero detuning between the SQD and the cavity mode, the coupled-system spontaneous emission can occur at either of two frequencies separated by $2 g$. If the coupled transition of the SQD is between excited state $|e\rangle$ and ground state $|g\rangle$ and the quantized field state with $n$ photons in the cavity mode is denoted by $|n\rangle$, then the two coupled-system eigenstates can be written as $|e 0\rangle_{ \pm}|g 1\rangle$. Since neither eigenstate can be written as a product of a quantum dot (QD) state and a cavity state, each has entanglement-a basic ingredient of quantum information science. Recently, there have been three experimental claims of observing this vacuum Rabi splitting using for the nanocavity a micropillar, ${ }^{6}$ a photonic

${ }^{a)}$ Electronic mail: jhendrickson@optics.arizona.edu crystal slab, ${ }^{7}$ and a microdisk. ${ }^{8}$ The photonic crystal slab nanocavity has the smallest mode volume, $(0.3-1) \lambda^{3}$. Therefore, it will have the largest vacuum Rabi splitting $2 g$ for a given SQD dipole moment, since $E_{\mathrm{vac}} \propto 1 / \sqrt{ } V$.

For many years, $\kappa$ of a photonic crystal nanocavity has been larger than $\gamma$; therefore, since $\kappa=2 \pi \nu / Q$, it has been $g / \kappa \propto Q / \sqrt{ } V$ that needed to be maximized for strong coupling. Even when $g / \kappa>1$ has been achieved in principle for a particular nanocavity and a given SQD dipole moment, one has the problem of finding a SQD that is accidentally situated spatially close to the intracavity field maximum within the tiny mode volume and accidentally positioned spectrally close to the cavity mode frequency. To increase the statistical likelihood of this occurring, it is tempting to make the QD density very high. But if it is too high, there are two undesirable consequences. The emission lines of so many QDs start to overlap, complicating the study of cavity coupling to one particular SQD. And the ensemble absorption of the layer of QDs situated throughout the semiconductor slab reduces the $Q$. When the QD density is low enough to avoid these problems, then the likelihood of finding vacuum Rabi splitting in any given nanocavity is very low. One must look for it by searching through many nanocavities, looking for the required accidental coincidences in space and frequency. In all three of the reported strong coupling experiments, the temperature was used to change the SQD/nanocavity detuning, taking advantage of the fact that the SQD transition shifts to a longer wavelength with increased temperature much faster than the cavity mode. Temperature scanning, however, is limited to $\cong 0.7 \mathrm{~nm}$ because of the increase in QD linewidth $\gamma$ due to phonon broadening at higher temperatures. A technique for changing the detuning while keeping the system at $\sim 4 \mathrm{~K}$ would minimize the phonon broadening. And if the technique could scan further than $1 \mathrm{~nm}$, it would make it easier to find the required coincidences at a given dot density, or it would make it possible to find them at a lower QD density. Even if one finds a way to grow a SQD in the 


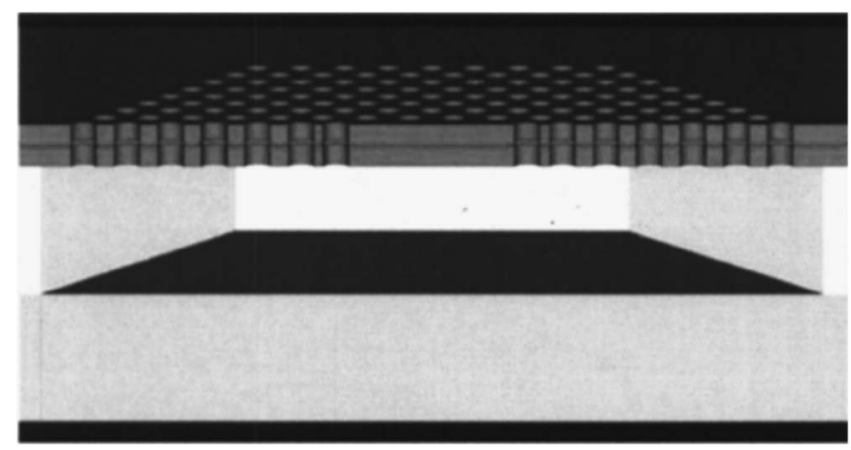

FIG. 1. A schematic of the photonic crystal slab nanocavity.

center of a photonic crystal nanocavity or to fabricate a photonic crystal nanocavity around a spatially isolated SQD, for the foreseeable future it will still be a challenge to make the SQD transition frequency coincide with the cavity frequency, i.e., scanning will still be essential. Here, we report on a $4 \mathrm{~nm}$ scan range achieved by condensing Xe gas onto a photonic crystal slab nanocavity held at $20 \mathrm{~K}$.

Figure 1 is a schematic of our photonic crystal slab nanocavity. Vertical optical confinement is by total internal reflection at both the top and bottom semiconductor/air interfaces. The stopband of the two-dimensional photonic crystal provides in-plane "mirrors." The cavity mode is created by omitting three holes to open up a defect state within the stopband. The shifting out of the "spacer" end holes greatly increases the $Q .{ }^{7,9}$ The distribution of the intracavity field shows that the field is still appreciable at the edges of the holes around the spacer and at the semiconductor/air interfaces where the Xe can condense. ${ }^{7}$ This explains why a large shift is observed even though there are no holes in the center of the spacer in which Xe can condense. Much larger shifts would be obtained using a nanocavity with the defect introduced by reducing the diameter of one hole. ${ }^{10}$ But for strong coupling applications, the larger spacer and larger $Q$ of the present design are essential, and the $5 \mathrm{~nm}$ scan range is still large enough to be very useful.

The shift experiments are performed as follows. The wavelength of a cavity mode is observed by detecting photoluminescence (PL) from a single layer of QDs with a density of $300-400 \mu \mathrm{m}^{2}$, grown by molecular beam epitaxy in the center of the $\sim 270 \mathrm{~nm}$ GaAs slab. A nanocavity was selected that has a mode around $1237 \mathrm{~nm}$, on the longwavelength edge of the QD ensemble PL; this avoids the reduction of $Q$ by the ensemble QD absorption. The QDs are optically pumped nonresonantly by focusing the output of a continuous-wave titanium-sapphire laser onto the nanocavity with a spot diameter of about $2 \mu \mathrm{m}$, using a reflective microscope objective with numerical aperture 0.5 . When the pump power exceeds roughly $100 \mu \mathrm{W}$, the uncoupled and very weakly coupled QDs with radiative lifetimes of 1-2 ns are almost all saturated. More strongly coupled QDs within the cavity peak are then able to emit many more photons per unit time, making the cavity peak stand out clearly. ${ }^{7,11}$ The $\mathrm{PL}$ is collected by the same objective in reflection geometry, dispersed in wavelength by a spectrometer, and detected by an InGaAs linear array. The sample is mounted in a Cryovac (Germany) Konti-Mikro cryostat with temperature control from 325 to $3.5 \mathrm{~K}$, by adjusting the He flow and/or the internal heater. The sample can be moved horizontally and vertically by a computer-controlled internal nanopositioner; 13000 for the first $3 \mathrm{~nm}$ before dropping slowly to 6000 .
Downloaded 14 Dec 2005 to 131.215 .225 .171 . Redistribution subject to AIP license or copyright, see http://apl.aip.org/apl/copyright.j
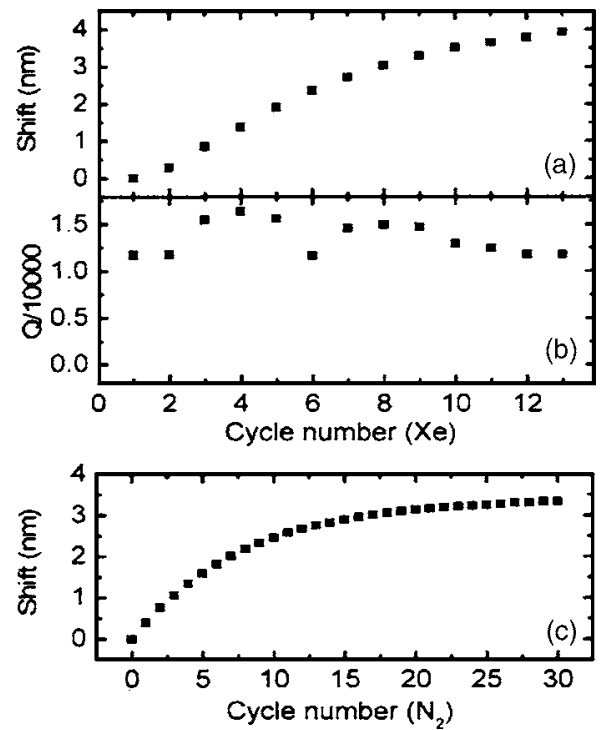

FIG. 2. The shift of cavity peak (a) and the value of the cavity quality factor $Q$ (b) as a function of the Xe condensation cycle $(0.5$ Torr of Xe was introduced each cycle in a 0.75 liter volume; the temperature was $20 \mathrm{~K}$ ). (c) Same as (a) for $\mathrm{N}_{2}(60$ Torr per cycle; $10 \mathrm{~K})$. The condensation time was approximately $1 \mathrm{~min}$, and the continuous-wave excitation was at $740 \mathrm{~nm}$.

this is critical for the stability of our measurements and for the ability to re-find a specific nanocavity.

The Xe gas (99.999\% pure) is admitted from a highpressure $(\sim 5000 \mathrm{kPa})$ tank, through a regulator that reduces the pressure to $\sim 30 \mathrm{kPa}$ and an ultrahigh-vacuum valve, into a vacuum chamber with a volume of $\sim 0.75$ liter. Both the admission line (including the regulator) and the vacuum chamber are initially evacuated to a pressure of $\sim 2 \times 10^{-6}$ Torr, to reduce the presence of impurities (air and water vapor). Another valve isolates the vacuum chamber from the cryostat where the sample is kept at low temperature. Once the desired pressure (measured with a convection gauge) of pure Xe gas is achieved in the vacuum chamber, the $\mathrm{Xe}$ flow is stopped. The $\mathrm{Xe}$ gas accumulated in the vacuum chamber is then allowed into the cryostat. The sample and all other sufficiently cold surfaces act as a very efficient adsorption pump, condensing the Xe gas. The shift of the cavity peak is essentially simultaneous with the Xe gas condensation, and it can be monitored live. After the initial pressure is recovered, the cycle is repeated.

For our particular system, there is a very narrow Xe pressure range that can be used. For a pressure below about 0.45 Torr, there is no shift even if the cycle is repeated many times. If the pressure is much above 0.5 Torr, the $\sim 4 \mathrm{~nm}$ scan limit is reached in one or a few cycles. However, in the last case, the quality factor $Q$ of the cavity is degraded, so very fast scanning has no practical value.

Some results from scanning experiments are shown in Fig. 2. The laser pump power was adjusted to $1.5 \mu \mathrm{W}$, to minimize laser-induced heating, and the temperature was held at $20 \mathrm{~K}$. For Xe, initially the cavity peak shifts quasilinearly with the amount of gas introduced, then the shift starts to saturate; see Fig. 2(a). $Q$, the quality factor of the cavity, starts with a value of $\sim 12000$ and does not drop below 11500 , as shown in Fig. 2(b). The behavior of $Q$ depends upon the particular nanocavity: Another one was scanned almost $5 \mathrm{~nm}$, with the $Q$ remaining constant at 13000 for the first $3 \mathrm{~nm}$ before dropping slowly to 6000 . 
If $\mathrm{N}_{2}$ is used instead of $\mathrm{Xe}$, holding the sample at a temperature of $20 \mathrm{~K}$, it is also possible to scan up to $4 \mathrm{~nm}$, but the results are much less reproducible. Lowering the temperature to $10 \mathrm{~K}$, the scanning becomes smooth and reproducible, but the scan limit is reduced to about $3.4 \mathrm{~nm}$ ( $2.7 \mathrm{~nm}$ without much degradation of $Q$ ), as shown in Fig. 2(c). Also, the amount of $\mathrm{N}_{2}$ gas used for condensation is more than 100 times that of Xe.

Of course, after scanning, one must remove the condensed gas before making a new scan. This is done by warming up the sample above the melting temperatures $(161.4 \mathrm{~K}$ for Xe and $63.3 \mathrm{~K}$ for $\mathrm{N}_{2}$ ), while continuously pumping on the system. Or, the cavity peak shifts back to its initial value due to local heating by a high-power $(\sim 0.5 \mathrm{~mW})$ laser beam, for sample temperatures above $20 \mathrm{~K}$. This effect occurs for $\mathrm{N}_{2}$, but not for Xe; it, as well as the behavior of the scanning above $20 \mathrm{~K}$, can be attributed to the lower melting point of nitrogen. Therefore, $\mathrm{N}_{2}$ is easier for multiple coarse scans, whereas Xe scans more precisely.

In fact, the idea of scanning by Xe condensation arose from lack of reproducibility of the cavity wavelength in earlier runs; we concluded this irreproducibility came from the condensation of air or water vapor due to an inadequate vacuum. Often one does not take great care with the vacuum in a cryostat when the sample is a microcavity or multiple quantum wells whose optical properties are mostly determined by layers well below the surface (which can be cleaned periodically to remove any condensation). But the photonic crystal is very sensitive to condensation, because the mode wavelength depends on both the slab thickness and the hole diameter. When we first cool down the sample, the wavelength of the cavity mode is not predictable within several $\mathrm{nm}$, presumably due to the condensation of background gas. In order to recover a reproducible (shortest) wavelength, the sample is heated back up to $310 \mathrm{~K}$ and then immediately cooled back down. We guess this drives off the offending condensate that then recondenses somewhere else. This procedure is performed before starting the condensation.

Figure 3 illustrates that the condensation technique can indeed be used to scan the SQD/nanocavity detuning through a strong coupling anticrossing. This technique should enable $4 \mathrm{~K}$ operation, thus minimizing quantum dot dephasing that degrades strong coupling.

In conclusion, condensation of xenon or nitrogen gas onto a photonic crystal slab nanocavity has been shown to be a useful way to scan the cavity mode by as much as $5 \mathrm{~nm}$ while maintaining the nanocavity at $\leqslant 25 \mathrm{~K}$.

The Tucson group thanks DARPA, NSF AMOP, NSF EPDT, and AFOSR, the Caltech group thanks the MURI
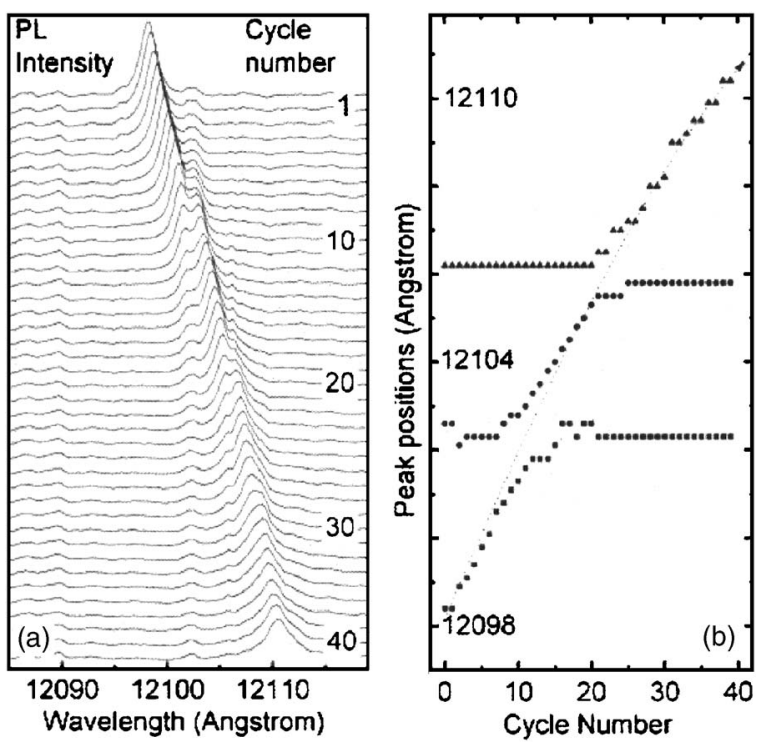

FIG. 3. Strong coupling anticrossings using Xe condensation to scan. (a) PL spectra at $25 \mathrm{~K}$ with low-power $(1.2 \mu \mathrm{W} ; 10 \mathrm{~s}$ exposure time $)$ continuouswave excitation at $770 \mathrm{~nm}$ as a function of detuning controlled by condensing $\mathrm{Xe}$ in increments of 0.46 Torr each cycle. Note that an uncoupled SQD transition energy does not shift with condensation. (b) Peak positions in the spectra vs cycle number. Two anticrossings (the signature of vacuum Rabi splitting strong coupling) occur between the nanocavity mode and two distinct SQDs. The dashed line shows the positions of the cavity peak for high-power (125 $\mu \mathrm{W} ; 0.2 \mathrm{~s}$ exposure time) excitation.

Center for Photonic Quantum Information Systems (ARO/ ARDA), NSF-ECS-NIRT, and AFOSR, and the Texas group thanks NSF-ECS-NIRT for financial support.

${ }^{1}$ E. Moreau, I. Robert, J. M. Gérard, I. Abram, L. Manin, and V. ThierryMieg, Appl. Phys. Lett. 79, 2865 (2001).

${ }^{2}$ M. Pelton, C. Santori, J. Vučković, B. Zhang, G. S. Solomon, J. Plant, and Y. Yamamoto, Phys. Rev. Lett. 89, 233602 (2002).

${ }^{3}$ J. Vučković, D. Fattal, C. Santori, G. S. Solomon, and Y. Yamamoto, Appl. Phys. Lett. 82, 3596 (2003).

${ }^{4}$ A. Imamoglu, Opt. Photonics News 13, 22 (2002).

${ }^{5}$ P. Michler, A. Kiraz, C. Becher, W. V. Schoenfeld, P. M. Petroff, L. Zhang, E. Hu, and A. Imamoglu, Science 290, 2282 (2000).

${ }^{6}$ J. P. Reithmaier, G. Sek, A. Löffler, C. Hofmann, S. Kuhn, S. Reitzenstein, L. V. Keldysh, V. D. Kulakovskii, T. L. Reinecke, and A. Forchel, Nature (London) 432, 197 (2004).

${ }^{7}$ T. Yoshie, A. Scherer, J. Hendrickson, G. Khitrova, H. M. Gibbs, G. Rupper, C. Ell, O. B. Scheckin, and D. G. Deppe, Nature (London) 432, 200 (2004)

${ }^{8}$ E. Peter, P. Senellart, D. Martrou, A. Lemaître, J. Hours, J. M. Gerard, and J. Bloch, quant-ph/0411076 (2004).

${ }^{9}$ Y. Akahane, T. Asano, B.-S. Song, and S. Noda, Nature (London) 425, 944 (2003).

${ }^{10}$ M. Loncar, A. Scherer, and Y. Qiu, Appl. Phys. Lett. 82, 4648 (2003).

${ }^{11}$ T. D. Happ, I. I. Tartakovskii, V. D. Kulakovskii, J.-P. Reithmaier, M. Kamp, and A. Forchel, Phys. Rev. B 66, 041303(R) (2002). 